\title{
Antagonistic pleiotropy may help population-level selection in maintaining genetic polymorphism for transmission rate in a model phytopathogenic fungus
}

\author{
A Tellier ${ }^{1}$, LMMA Villaréal and T Giraud \\ Ecologie, Systématique et Evolution, UMR 8079 CNRS-UPS, Université Paris-Sud, Orsay, France
}

\begin{abstract}
It has been shown theoretically that the conditions for the maintenance of polymorphism at pleiotropic loci with antagonistic effects on fitness components are rather restrictive. Here, we use a metapopulation model to investigate whether antagonistic pleiotropy could help maintain polymorphism involving common deleterious alleles in the phytopathogenic fungus Microbotryum violaceum. This fungus causes anther smut disease of the Caryophyllaceae. A previous model has shown that the sex-linked deleterious alleles can be maintained under a metapopulation structure, when intra-tetrad selfing (mating between products of the same meiosis) is high, due to founder effects and selection at
\end{abstract}

the population level. Here, we add two types of pleiotropic advantages to the metapopulation model. A competitive advantage for strains carrying the sex-linked deleterious alleles did not facilitate their maintenance because competitive situations were too rare. In contrast, higher spore production did facilitate the maintenance of the deleterious alleles at low intra-tetrad mating rates and with a large advantage for spore production. These results show that antagonistic pleiotropy may promote the persistence of genetic variation, in combination with other selective forces. Heredity (2007) 98, 45-52. doi:10.1038/sj.hdy.6800902; published online 4 October 2006

Keywords: group selection; parasites; virulence; disease transmission; infectivity; protected polymorphism

\section{Introduction}

The maintenance of genetic diversity is a central problem in evolutionary genetics. The idea that polymorphism may be maintained by antagonistic pleiotropy (when mutations with deleterious effects on some characters have beneficial effects on other characters) (Rose, 1982) is popular and appealing, even though, initially Rose (1982) and more recently Curtsinger et al. (1994) and Hedrick (1999) have shown that conditions for polymorphism at pleiotropic loci with antagonistic effects on fitness are rather restrictive. Therefore, even if trade-offs in fitness components have been reported to occur in nature (e.g. Rose and Charlesworth, 1981), it is now considered unlikely that antagonistic pleiotropy can be an important factor in maintaining polymorphism. The alternative mechanisms proposed to protect polymorphism are frequency-dependent selection (advantage of rare alleles, for instance, in self-incompatibilities (Richman, 2000), overdominance (heterozygote advantage; Chotivanich et al., 2002), heterogeneous environments (Kisdi and

Correspondence: Dr T Giraud, Ecologie, Systématique et Evolution, UMR 8079 CNRS-UPS, Bâtiment 362, Université Paris-Sud, 91405 Orsay cedex, France.

E-mail: Tatiana.Giraud@ese.u-psud.fr

${ }^{1}$ Current address: Department of Disease and Stress Biology, John Innes Centre, Colney, Norwich NR4 7UH, UK.

Received 2 January 2006; revised 2 August 2006; accepted 24 August 2006; published online 4 October 2006
Geritz, 1999), different levels of selection (Couvet et al., 1998; Johnson and Boeriijst, 2002; Tellier et al., 2005) or temporal fitness variability (Felsenstein, 1976; Prout and Savolainen, 1996).

Here, using a metapopulation model, we investigate whether different types of pleiotropic advantages could facilitate, in conjunction with other selective forces, the maintenance in a polymorphic state of the sex-linked deleterious alleles that have been reported to occur at relatively high frequencies in the phytopathogenic fungus Microbotryum violaceum (Kaltz and Shykoff, 1997; Oudemans et al., 1998; Thomas et al., 2003). The anther-smut fungus $M$. violaceum is a basidiomycete obligate parasite of many Caryophyllaceae (Thrall et al., 1993). The disease sterilizes, but it does not kill its host plant after producing teliospores in the place of pollen. Teliospores are transported from diseased to healthy plants by pollinators (Roche et al., 1995; Shykoff and Bucheli, 1995). Once deposited on a host plant, diploid teliospores undergo meiosis and give rise to four haploid cells (two of mating type A1 and two of mating type A2). Each of these cells buds off saprophytic yeast-like sporidia on the plant surface. New infectious dicaryons are rapidly produced by conjugation of two sporidia of opposite mating type. M. violaceum can perform either outcrossing or selfing (Baird and Garber, 1979; Giraud et al., 2005, 2006), and selfing can occur either between cells from different tetrads of the same fungal individual (inter-tetrad conjugation) or between products of a single 
meiosis (intra-tetrad conjugation; Hood and Antonovics, 2000). Dicaryotic hyphae then enter the host tissue and grow along with the plants' apical meristematic regions. Infection is systemic and plants produce only diseased flowers (Thrall et al., 1993).

Several studies (Garber et al., 1978; Kaltz and Shykoff, 1997; Oudemans et al., 1998; Thomas et al., 2003) have shown that some $M$. violaceum strains produce teliospores, all giving rise to sporidia of only one mating type. This individual mating-type-ratio distortion is due to the presence of deleterious alleles at haploid phase, 'haplolethals', linked to one mating type (Hood and Antonovics, 2000). These strains with a mating-type-ratio distortion (hereafter called 'biased strains') still produce four cells following meiosis, but sporidia carrying the haplo-lethal allele quickly die after a few mitotic divisions (Hood and Antonovics, 2000).

Teliospores of a strain with a biased mating-type ratio are still fully able to produce an infectious dicaryon by early intra-tetrad conjugation (Hood and Antonovics, 2000): conjugation takes place between products of a single tetrad, just after meiosis, before the death of the cells carrying haplo-lethal alleles. Using controlled crosses (Baird and Garber, 1979; Giraud et al., 2005; Giraud et al., 2006), estimations of intra-tetrad mating rates fell between 0.43 and 0.89 , with some genetic or environmental variability. Biased strains are expected to suffer from infection disadvantages. For a given number of teliospores, unbiased strains will indeed produce many more conjugating dicaryons than biased strains: all teliospores not performing intra-tetrad mating will yield no dicaryon in biased strains (when alone on a plant), whereas they will produce many conjugating dicaryons in unbiased strains. Infection probability has indeed been shown to be lower for biased strains than for non-biased strains in single-strain artificial inoculations (Giraud et al., 2006).

Three hypotheses have been proposed to account for the persistence at high frequencies of mating-type-linked haplo-lethal alleles in natural populations. First, Antonovics et al. (1998) showed, using a model of an infinite panmictic population of $M$. violaceum, that mating-typelinked haplo-lethal alleles could persist only if they exhibited a diploid advantage. This hypothesis considers that haplo-lethal mutations may result in more rapid or efficient growth within the host, leading, for instance, to a higher spore production. Using artificial inoculations, Giraud et al. (2006) indeed found that biased strains had a higher spore production, although they suggested that the timing may render the increase in spore production irrelevant. The previous model based on antagonistic pleiotropy (Antonovics et al., 1998), however, cannot allow the persistence of the haplo-lethals in a polymorphic state in a finite population.

A second hypothesis is that biased strains could have a competitive advantage against unbiased strains, in terms of rapidity of infection. Thomas et al. (2003) proposed that two infection strategies could exist: production of numerous sporidia giving many dicaryons and a high infection probability, or alternatively quick intrapromycelial conjugation, with a lower infection probability, but a time advantage in competition with the first strategy. In populations polymorphic for these two strategies, haplolethal alleles would be neutral in individuals with the second strategy. Haplo-lethals could then be linked to the rapid strategy, and have an advantage when in competition with the first strategy that can only be present in unbiased strains. This rapid strategy is expected to be at a greater competitive advantage as the prevalence of disease increases, as in heavily diseased populations, plants are more likely to receive spores from different genotypes simultaneously. Because prevalence varies among natural populations of Silene latifolia (Thomas et al., 2003), such an advantage of biased strains would be spatially heterogeneous and could, therefore, maintain polymorphism. Competition situations are, however, expected to be rare in this system, because diseased plants are rarely visited (Jennersten, 1988; Shykoff and Bucheli, 1995), and it is, therefore, not trivial whether such a competitive advantage can indeed maintain polymorphism at loci with haplo-lethal alleles. Conflicting experimental results have been obtained regarding such a competitive advantage of biased strains: using different protocols of mixed experimental inoculations, Hood (2003) found that biased strains were better at infecting than unbiased strains, whereas Giraud et al. (2006) found the reverse.

A third hypothesis is that haplo-lethals are maintained in metapopulations without pleiotropic advantage due to founder effects and selection at the population level. A model based on the assumption that biased strains had a constantly lower transmission rate than non-biased strains showed that populations founded by biased strains may experience a lower extinction rate than populations founded by non-biased strains, precisely because of their lower infection ability: strains carrying haplo-lethals should spread slower, allowing their host population to reproduce more and, therefore, to be less prone to extinction (Tellier et al., 2005). Haplo-lethals could, however, be maintained only with high intratetrad mating rates (higher than approximately 0.7 ).

The aim of this study was to investigate the effect of pleiotropic advantage of mating-type-linked haplolethals in metapopulation of $M$. violaceum infecting S. latifolia. We, therefore, used our previous model of a metapopulation of $M$. violaceum (Tellier et al., 2005), adding two types of pleiotropic advantages: higher probability of infection by biased strains in competition situations and higher spore production by biased strains. We examined analytically and through simulations whether, and under which conditions, each of these two types of pleiotropic advantages can facilitate the maintenance of haplo-lethals.

\section{The model}

We used the spatially explicit simulation model of sets of local populations (= patches) described in Tellier et al. (2005). Each generation, within-patch dynamics of disease transmission, was first simulated through deterministic equations, then a percentage of seeds and teliospores of each occupied site was allowed to disperse stochastically and thirdly, some patches went extinct stochastically.

\section{Within-patch dynamics}

Sexually transmitted diseases, including $M$. violaceum disease on S. latifolia with pollinators for vectors, are characterized by frequency-dependent transmission within populations (Getz and Pickering, 1983). Because 
diseased plants are rarely visited (Jennersten, 1988; Shykoff and Bucheli, 1995), we considered that vectors were likely to carry fungal teliospores from only the two previously visited hosts, if diseased.

In a given population at time $t+1$, the numbers of healthy plants $\left(X_{t+1}\right)$, of plants infected by a biased strain $\left(Y_{t+1}\right)$ and of plants infected by an unbiased strain $\left(Z_{t+1}\right)$ can be written as follows:

$$
\begin{aligned}
X_{t+1}=X_{t} *[b+ & (1-d) \\
* & \left(1-Y_{t}\left(\beta_{\mathrm{B}} \frac{\phi \alpha}{N_{t}}+\beta_{\mathrm{B}} \phi \alpha^{2} \frac{Y_{t}}{N_{t}^{2}}+\beta_{\mathrm{C}} \Delta_{\mathrm{B}} \alpha^{2} \frac{Z_{t}}{N_{t}^{2}}\right)\right. \\
- & \left.\left.Z_{t}\left(\beta_{\mathrm{NB}} \frac{\alpha}{N_{t}}+\alpha^{2} \beta_{\mathrm{NB}} \frac{Z_{t}}{N_{t}^{2}}+\beta_{\mathrm{C}} \Delta_{\mathrm{NB}} \alpha^{2} \frac{Y_{t}}{N_{t}^{2}}\right)\right)\right] \\
Y_{t+1}= & (1-d) *\left(Y_{t}+\beta_{\mathrm{B}} \phi \alpha \frac{Y_{t}}{N_{t}} X_{t}\right. \\
& \left.+\beta_{\mathrm{B}} \phi \alpha^{2} \frac{Y_{t}^{2}}{N_{t}^{2}} X_{t}+\Delta_{\mathrm{B}} \beta_{\mathrm{C}} \alpha^{2} \frac{Y_{t} Z_{t}}{N_{t}^{2}} X_{t}\right) \\
Z_{t+1}= & (1-d) *\left(Z_{t}+\beta_{\mathrm{NB}} \alpha \frac{Z_{t}}{N_{t}} X_{t}\right. \\
& \left.+\beta_{\mathrm{NB}} \alpha^{2} \frac{Z_{t}^{2}}{N_{t}^{2}} X_{t}+\Delta_{\mathrm{NB}} \beta_{\mathrm{C}} \alpha^{2} \frac{Z_{t} Y_{t}}{N_{t}^{2}} X_{t}\right)
\end{aligned}
$$

where $d$ is the intrinsic death rate $(d=0.1$, Thrall et al., 1995), $\alpha$ is the probability that a pollinator visiting a healthy flower leaves spores of $M$. violaceum $(\alpha=0.25), b$ is the host birth rate and $N_{\mathrm{t}}$ is the total host population at generation $t . \beta_{\mathrm{NB}}$ denotes the infection probability of unbiased strains when alone on a plant, $\beta_{\mathrm{B}}$ the infection probability of biased strains when alone on a plant, and $\beta_{\mathrm{C}}$ is the global infection probability when biased and unbiased strains are in competition on the same host plant. $\Delta_{\mathrm{NB}}$ is the proportion of competition events won by unbiased strains and $\Delta_{\mathrm{B}}$ the proportion of competition events won by biased strains.

We assumed a density-dependent per capita reproductive rate of S. latifolia, and the host birth rate $b$ was written as follows:

$$
b=\frac{\lambda}{\gamma N_{t}+1}
$$

where $\lambda$ is the maximum reproductive rate of the host $(\lambda=2$; Thrall and Jarosz, 1994b) and $\gamma$ is a constant that determines the strength of the density dependence $(\gamma=0.4$; Thrall and Jarosz, 1994b).

In Equations (2) and (3), we considered three types of newly infected plants in each patch. First, there were plants that received spores from only one diseased plant (second term in brackets). Second, there were plants that received spores from two diseased plants, but all spores were of the same type (biased or unbiased) (third term in brackets). Third, there were plants that received spores from two diseased plants, one with biased spores and the other with unbiased spores (fourth term in brackets). To determine which type of spores won if infection was successful in this latter case, we assumed that the plants received a panmictic population of biased and unbiased strains, and we applied the formula from the panmictic model of Antonovics et al. (1998):

$$
U_{t+1}=U_{t} \frac{\phi+(2(1-\phi)) /\left(U_{t}+1\right)}{\phi+\left(2 U_{t}(1-\phi)\right) /\left(U_{t}+1\right)}
$$

$U_{t}$ being the proportion of unbiased strains deposited on plants in a competition situation at the beginning of generation $t$ and $\phi$ the fraction of biased strains that undergoes intra-tetrad selfing. The value of $U_{t}$ depends on the sporulation advantage of the biased strains. $\Delta_{\mathrm{NB}}$ the proportion of competition events won by unbiased strains (respectively $\Delta_{\mathrm{B}}$ for biased strains) is, therefore, written as

$$
\begin{aligned}
& \Delta_{\mathrm{NB}}=\eta * U_{t+1} \\
& \Delta_{\mathrm{B}}=(1-\eta) *\left(1-U_{t+1}\right)
\end{aligned}
$$

where $\eta$ is a competitive factor $(\eta=0.5$ if no advantage is given to either type of strain in competition, and $\eta>0.5$ if unbiased strains have an advantage over biased strains in competitive situations).

We considered earlier that pollinators can carry spores from only two diseased plants at a time although vectors may contact three (or more) infected hosts. The likelihood of such event is the product of all three (or more) diseased host frequencies multiplied by the probability of depositing three (or more) times spores on a plant ( $\alpha$ elevated to the power three or higher). The likelihood of such multiple contacts is a negligible probability and, therefore, does not affect the disease dynamics driven mainly by coefficients of lower order (single and double vector contacts; Equations (1), (2), (3)).

\section{Among-patch dynamics (Figure 1)}

The metapopulation was composed of a one-dimensional array of 200 patches with two extreme absorbing boundaries, to mimic linear roadside metapopulations of S. latifolia (Antonovics et al., 1994; Thrall and Jarosz, 1994a, b). Strong genetic differentiation has been found among plant populations, suggesting that plant dispersal is limited (Delmotte et al., 1999). In the model, a fraction $m_{\mathrm{H}}=0.05$ of seeds disperse to the four neighbouring patches according to a Weibull probability distribution with a probability $P(i)$ of landing in a patch distant by $i$ units from the source patch:

$$
P(i)=\frac{\kappa}{\theta^{\kappa}} i^{\kappa-1} \mathrm{e}^{-(i / \theta)^{\kappa}}
$$

where the parameters $\kappa$ and $\theta$ control, respectively, the scale and the shape of the dispersal curve $(\kappa=0.9$ and $\theta=0.7$; Thrall and Jarosz, 1994b; Tellier et al., 2005). We assume here that the distance between patches is constant, as was done in previous models of this system (Thrall and Jarosz, 1994a; Thrall et al., 1995). Changing the distance between patches affects only the speed of colonization of the plant metapopulation (not shown).

Metapopulations of $M$. violaceum have been shown to follow an island model dynamics where no isolation by distance can be detected among populations (Delmotte et al., 1999; Giraud, 2004). In our model, a fraction $m_{\mathrm{P}}=0.125$ of the diseased plants in a given patch send teliospores towards any of the other occupied patches, 


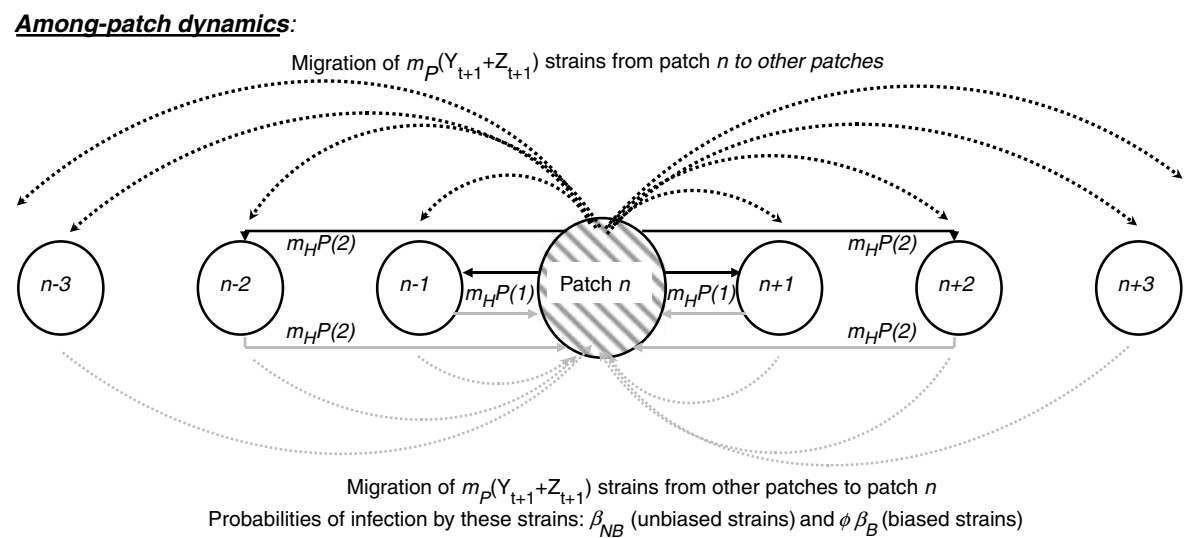

Figure 1 Schematic representation of the among-patch dynamics of the model (see text for details). The solid lines represent the steppingstone seed migration to the four neighbouring patches and the dotted lines represent the $M$. violaceum spore dispersal to any other patch. Spore dispersal from the focal patch (patch $n$ ) to other adjacent patches is represented in black, and dispersal from adjacent patches to the focal patch $n$ is represented in grey $\left(m_{\mathrm{H}}=0.05, m_{\mathrm{P}}=0.125, P(1)\right.$ and $P(2)$ given in Equation (8)).

where they infect unbiased and biased strains with probabilities of $\beta_{\mathrm{NB}}$ or $\beta_{\mathrm{B}} * \phi$, respectively.

After among-patch dispersal, probability of extinction is calculated for each occupied patch depending on the total number of plants in a patch $\left(N_{\mathrm{t}}\right)$, and the extinction coefficient $K_{\text {ext }}=0.9$. The extinction probability associated to a given occupied patch is therefore:

$$
P\left(N_{t}\right)=1 /\left(1+N_{t} * K_{\mathrm{ext}}\right)
$$

\section{Initialization}

To start each simulation, each of 40 patches out of the 200 possible were randomly filled with $N_{0}=50$ plants. Of these initial populations, $20 \%$ were then randomly chosen to be diseased at a prevalence of 0.25 . Each initial fungal population had a proportion $M_{0}=0.5$ of unbiased strains. Each simulation was allowed to run for 400 generations, by which spatial and temporal patterns generally became stable.

\section{Parameter values for the different models}

Model A of sporulation advantage of haplo-lethals assumed that (i) in non-competition situations, more biased than unbiased teliospores were present on plants and (ii) in competition situations, biased teliospores outnumbered unbiased teliospores. Model A was, therefore, characterized by higher values of infection probability for the biased strains $\left(\beta_{\mathrm{B}}>0.4\right)$ and higher proportions of biased strains in competition situations $\left(U_{t}<0.5\right)$.

Model B assumed that biased strains were more fit than unbiased strains in competition. When competition between the two types of strains occurred, the proportions of biased and unbiased strains remained equal $\left(U_{t}=0.5\right)$, but the competitive factor $\eta$ gave an advantage to biased strains $(\eta<0.5)$.

The effect of pleiotropic advantages on biased strain maintenance was studied for the whole range of values of intra-tetrad selfing rates $(\phi)$. The following results focus on rates lower than 0.7 because they did not allow the maintenance of haplo-lethals without diploid advantage (Tellier et al., 2005).

\section{Modelling and statistical analyses}

Thirty simulations for each set of parameters values were performed using the Scilab software (v 3.1; Copyright INRIA 2004; France), and this appeared to be sufficient given the low variances in results among replicates. GenStat for Windows (8th Edition, VSN International, Oxford, UK) was used to test the significance of mean differences by multiple comparison tests (NewmanKeuls and Bonferroni tests; Zar, 1984).

\section{Results}

\section{Effect of a sporulation advantage associated to haplo-lethals}

Model A examined whether a sporulation advantage of biased strains improved the maintenance of haplolethals. We first derived analytically the compensation values of the infection probability of biased strains $\beta_{\mathrm{B}}$ and of the proportion of biased spores $U_{t}$ that exactly cancelled out the disadvantage of biased strains in a single panmictic population.

Considering first competition situations, a higher sporulation rate of biased strains resulted in a higher relative proportion of biased spores $U_{t}$. The compensation value of the proportion of biased strains in competition situations $\left(U_{\mathrm{C}}\right)$ can be calculated from equation (5):

$$
-2 \phi U_{\mathrm{C}}^{2}+U_{\mathrm{C}}(\phi-2)+\phi=0
$$

hence

$$
U_{\mathrm{C}}=\frac{\phi-2 \pm \sqrt{9 \phi^{2}-4 \phi+4}}{4 \phi}
$$

Considering the strains arriving alone on a healthy plant, a higher sporulation of biased strains translates into better infection probability, because the probability of infection is linked to the number of dicaryons produced (Roche et al., 1995; Kaltz and Shykoff, 1999). The compensation value of the infection probability parameter $\beta_{\mathrm{B}}$ is $\beta_{\mathrm{B}}=\beta_{\mathrm{NB}} / \phi$. Smaller intra-tetrad selfing rates $(\phi)$ thus require proportionally higher compensation 
values of the infection probability parameter, that is, greater sporulation advantages of biased strains.

To explore the fate of haplo-lethals for values of the parameters $U_{t}$ and $\beta_{\mathrm{B}}$ below those of compensation, we introduced a parameter $D c$ representing the degree of compensation. $D c$ measures the sporulation advantage of biased strains versus unbiased strains: $D c=0$ if no advantage is given to biased strains, and $D c=1$ if $U_{t}$ and $\beta_{\mathrm{B}}$ are at their compensation values. We used in simulations the parameters $U_{t}$ and $\beta_{\mathrm{B}}$ as functions of $D c$ :

$$
U_{t}=U_{\text {base }}-\left(U_{\text {base }}-U_{\mathrm{C}}\right) * D c \quad \text { and }
$$

$$
\beta_{\mathrm{B}}=\beta_{\mathrm{NB}} *(1+D c(-1+1 / \phi))
$$

where $U_{\text {base }}=0.5$ and $\beta_{\mathrm{NB}}=0.4$ are the values of the sporulation parameters without pleiotropic advantage.

As expected, when $D c=1$, equal proportions of biased and unbiased strains were maintained in a single infinite panmictic population (Figure 2) and in metapopulations (Figure 3). For degrees of compensation less than 1, even slightly, biased strains rapidly vanished in a single panmictic population (Figure 2). Biased strains then produced more spores than unbiased strains, but this did not fully compensate for their smaller production of infectious dicaryons. In contrast, biased strains could persist in a stable polymorphic state under metapopulation dynamics even for degrees of compensation less than 1 (Figure 3). For instance, biased strains could be maintained at frequencies significantly different from 0 for $D c \geqslant 0.5$ when $\phi=0.5$, and for $D c \geqslant 0.4$ when $\phi=0.6$ (Figure 3, Newman-Keuls statistical groups, $P=0.01$ ). $D c \geqslant 0.5$ when $\phi=0.5$ means that spore production of biased strains is at least 1.5 times more than that of the unbiased strains (Equation (13)). For $\phi=0.6$, an advantage of at least 1.27 in spore production is required (Figure 3 and Equation (13)). Increasing Dc significantly increased haplo-lethal frequencies in the metapopulation (Figure 3, Newman-Keuls statistical groups, $P=0.01$ ).

For degrees of spore production advantage below 1, biased strains had a disadvantage in infection ability, as shown by their vanishing from a panmictic population (Figure 2). However, this disadvantage was compensated by population-level selection, a specific feature of metapopulation dynamics: populations with a majority of biased strains experienced lower extinction rates than

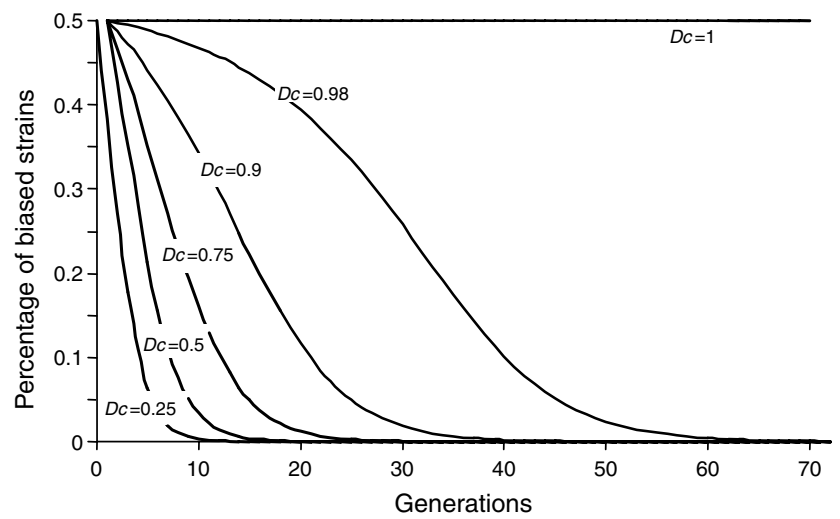

Figure 2 Change in the percentage of biased strains in a single panmictic population for different values of spore production advantage, $D c$, for an intra-tetrad selfing rate $\phi$ of 0.5 . populations with a majority of unbiased strains, because they sterilize their host population less efficiently. The extinction rate of populations having a majority of biased strains indeed increased with $D c$ (Figure 4), but remained significantly less than that of populations having a majority of unbiased strains (Figure 4, $0.9>D c>0.5$, groups of Newman-Keuls test, $P=0.01$ ). The sporulation advantage reduced the disadvantage of biased strains within populations, allowing their interpopulation advantage of lower population extinction rate to create a dynamic equilibrium. For $\phi \leqslant 0.7$, some degree of pleiotropic advantage in spore production $(D c \geqslant 0.5$ when $\phi=0.5$ for instance) thus helped population-level selection in maintaining biased strains in a polymorphic state.

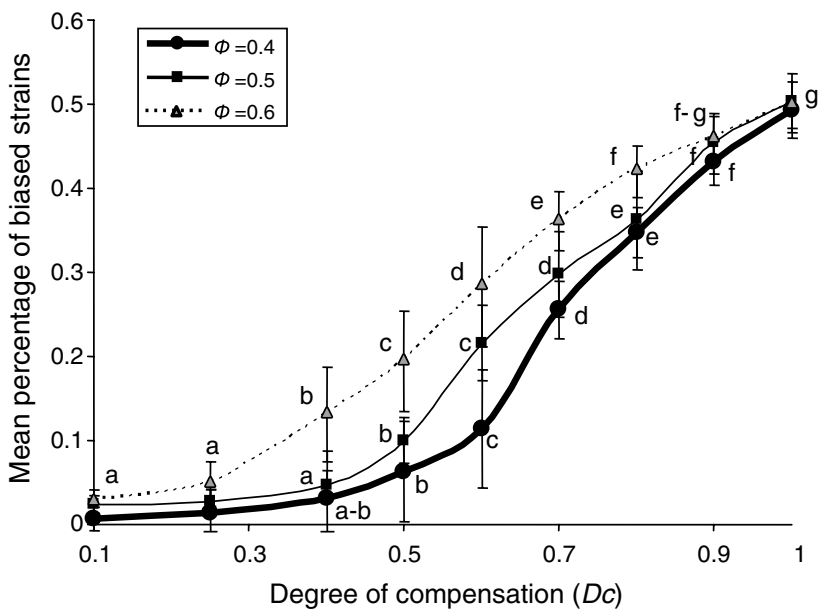

Figure 3 Percentage of biased strains in a metapopulation (mean \pm s.e. over populations and 30 simulations) for different values of spore production advantage, $D c$, and of intra-tetrad selfing rates $\phi\left(M_{0}=0.5\right.$; generation 400$)$. Different letters indicate significantly different groups of means with the multiple comparison Newman-Keuls test $(P=0.01)$, tests being carried out separately for each value of $\phi$.

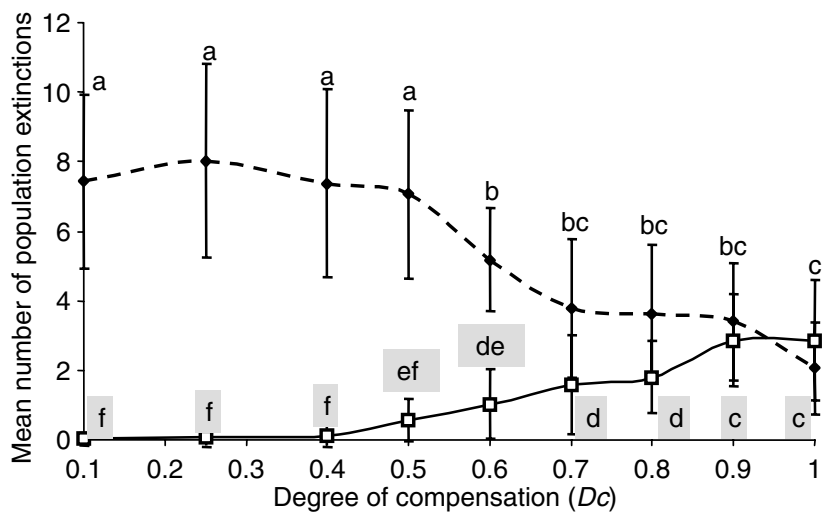

Figure 4 Number of extinct populations (mean \pm s.e. over 30 simulations) in a metapopulation for different values of spore production advantage, $D c$, for an intra-tetrad selfing rate $\phi$ of 0.5 $\left(M_{0}=0.5 ; 30\right.$ repetitions, generation 400$)$. The dotted line represents populations of unbiased strains and the solid line represents populations of biased strains. Different letters indicate significantly different groups of means with the multiple comparison NewmanKeuls test $(P=0.01)$ : top letters are for unbiased strains and bottom letters in grey boxes are for biased strains. 
Effect of competition advantage associated

to haplo-lethals

Model B considered that biased strains had a compensatory advantage when in competition against unbiased strains, for instance, in terms of rapidity of infection. We derived the compensation values of the competition parameter $\eta$, which offset the disadvantage of biased strains against unbiased strains when in competition on a host plant. This situation is expressed by $\Delta_{\mathrm{B}}=\Delta_{\mathrm{NB}}$, leading to the condition

$$
\eta=\frac{3 \phi}{4+2 \phi}
$$

When the competitive factor $\eta$ was less than the compensation value, biased strains had an infection advantage when in competition against unbiased strains, but they still had an infection disadvantage when alone on a plant compared with unbiased strains. Simulations were, therefore, needed to assess the influence of such a competitive advantage on the maintenance of biased strains in the metapopulation. We not only let the competitive factor $\eta$ vary in the simulations, but also the parameters affecting the occurrence of competition situations: the global infection probability in competition situations $\left(\beta_{\mathrm{C}}\right)$ as well as the probability of spore deposition by a pollinator $(\alpha)$.

Biased strains can be maintained in a single infinite panmictic population only if their frequency in the population is equal to that of unbiased strains (equality of Equations (2) and (3)). Moreover, even when biased strains exhibit the largest possible advantage in competition situations against unbiased strains $\left(\eta=0.01, \beta_{\mathrm{C}}=1\right.$ and $\alpha=0.5$ ), haplo-lethals can persist only in a single infinite panmictic population if the intra-tetrad selfing rate $(\phi)$ is higher than 0.87 (derivations from Equations. (2) and (3)).

We performed metapopulation simulations to investigate whether a competition advantage could help population-level selection in maintaining haplo-lethals for values of the intra-tetrad selfing rate for which they could not be maintained without pleiotropic advantage ( $\phi \leqslant 0.7$; Tellier et al., 2005), as spore production advantage did. Even when biased strains exhibited the largest possible advantages in competition situations against unbiased strains, biased strains nonetheless vanished from the metapopulation (Figure 5). Competition situations were in fact too rare for a competitive advantage

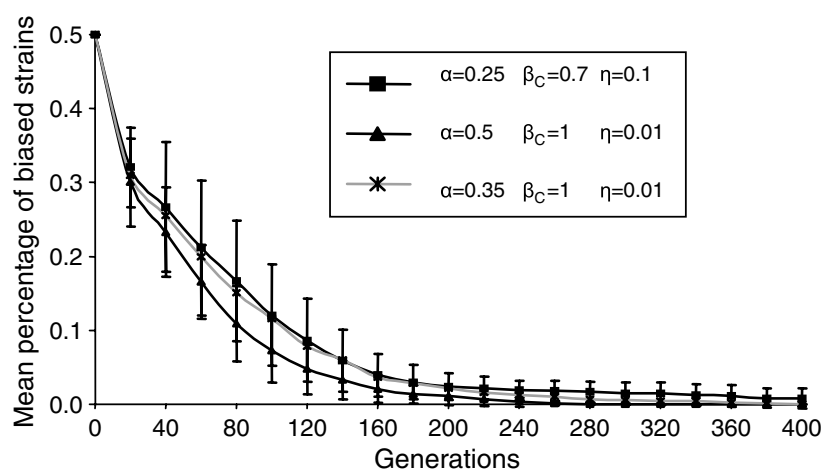

Figure 5 Change in the mean percentage of biased strains in a metapopulation (mean \pm s.e. over populations and 30 simulations) for different values of competition. of biased strains to have a significant effect on their maintenance.

\section{Robustness of the results}

Our model was run using the range of parameter values available from previous field studies (Thrall and Jarosz, 1994a, b; Thrall et al., 1995) because our aim was to be as realistic as possible. Some parameters, however, may be variable in natural conditions. so we investigated how changing parameter values affected the results.

The key parameters of the plant metapopulation dynamics are among population dispersal of plants $(\kappa$, $\left.\theta, m_{\mathrm{H}}\right)$, population extinction rates $\left(K_{\text {ext }}\right)$, and epidemiological parameters such as the host death rate $(d)$ and host birth rate $(b)$. Varying these parameters influenced the rate of colonization of healthy populations by the fungus and the rate of the establishment of new, healthy plant populations, thereby impacting strongly on the stability of the metapopulation, as shown in Tellier et al. (2005). However, all values allowing stable metapopulations (Tellier et al., 2005) gave similar results on the pleiotropic advantages required for haplo-lethal maintenance (not shown).

The second group of key parameters influenced disease spread within populations and thus disease prevalence. These parameters include the probability that a pollinator visiting a healthy flower leaves spores of $M$. violaceum $(\alpha=0.25)$ and the rate of spore dispersal $\left(m_{\mathrm{P}}=0.125\right)$. Changing the probability that a pollinator visiting a healthy flower leaves spores of $M$. violaceum $(\alpha)$ affected both metapopulation sustainability and haplolethal maintenance (Tellier et al., 2005). The value of $m_{\mathrm{P}}=0.125$ used in the simulations presented above was chosen to maximize coexistence of plants and fungi. Higher values of $m_{\mathrm{P}}\left(0.125<m_{\mathrm{P}}<0.25\right)$ did not lead to significant differences in spore production advantages required for haplo-lethal maintenance (for a given selfing rate $\phi$, Figure 2), but the metapopulation was less stable. At even higher values of $m_{\mathrm{P}}(>0.25)$, the metapopulation as a whole crashed in the simulations because the load of pathogen infection on plant populations was too high compared with their reproductive rate. Persistence of the metapopulation was never possible when $m_{\mathrm{P}}$ was higher than 0.25 (not shown).

In conclusion, the results obtained with the limited range of parameters extensively studied seem to be robust. When parameter values allow the coexistence of plants and pathogens, they produce similar results, in particular, regarding the sporulation advantage required for haplo-lethal maintenance. The threshold values for the advantage of spore production should not, however, be taken too strictly because it still could vary a little, depending on parameter values such as the spore migration rate $\left(m_{\mathrm{P}}=0.125\right)$, a key parameter for persistence of the metapopulation (not shown).

\section{Discussion}

In this study, we introduced pleiotropic advantages for haplo-lethals to investigate their effects on biased strain maintenance in a metapopulation. Model B explored the impact of an advantage of infection of biased strains in competition situations. Even when biased strains always outcompeted unbiased strains when in competition on the same plant, this effect did not improve the 
maintenance of haplo-lethals compared with the model without pleiotropic advantage (Tellier et al., 2005). Competition events between the two types of strains were too infrequent, in a single panmictic population as well as in metapopulation, for this advantage to have an effect on the maintenance of biased strains, even for the highest possible occurrence of competition situations in our model (highest values of vector contact rate, $\alpha$, infection probability in competition, $\beta_{\mathrm{c}}$ and competitive factor, $\eta$ ). In our model, pollinators could carry spores only from the two previously visited plants (if diseased). There is a lack of data on the frequency of competition situations in nature even if the scarce evidence suggests that this frequency should indeed be low. Hood (2003) showed that even a slight time advantage of a given strain of $M$. violaceum when arriving on a plant can determine its success of infection when in competition. True competition for infection must, therefore, essentially occur only when a plant is visited by a pollinator carrying the two types of strains at the same time. This is likely to be rare as pollinators exhibit a strong preference for healthy plants (Jennersten, 1988; Shykoff and Bucheli, 1995). Furthermore, within-population distributions of M. violaceum spores (Roche et al., 1995) and genotypes (Giraud, 2004) reflect strongly the leptokurtic dispersion, suggesting that mixtures of strains may rarely be deposited on healthy plants. The results of model B imply that it would not be useful to search for an advantage of biased strains in competition situations anyway, and that such advantage, as the slight withinhost competitive advantage of strains with a biased sexratio reported by Hood (2003), would be irrelevant to understand haplo-lethal maintenance. More data on the number of fungal genotypes deposited on healthy plants are, however, needed.

In contrast, sporulation advantages did permit significant improvement in biased strain maintenance: in the cases where population-level selection could not maintain biased strains by itself, this antagonistic pleiotropic advantage allowed their persistence in some cases, although it also was not sufficient by itself. The required sporulation advantage needs to be significantly high. For instance, when half of the sporidia are involved in inter-tetrad mating $(\phi=0.5)$, the sporulation rate of biased strains has to be 1.5 times as that of unbiased strains to allow maintenance of haplo-lethals.

Estimations of intra-tetrad mating rates from controlled crosses ranged between 0.43 and 0.89 (Baird and Garber, 1979; Giraud et al., 2005, 2006), with some genetic and/or environmental variability. These estimations include the range of values of intra-tetrad mating allowing the maintenance of haplo-lethal alleles even without diploid advantage, thanks to the populationlevel selection. Some estimates are, however, well below the 0.7 threshold, suggesting that a pleiotropic advantage may be needed to maintain haplo-lethals. Giraud et al. (2006) and ME Hood (personal communications), in fact, observed that biased strains had a higher spore production than unbiased strains. The difference was small (1.13 times more spores produced by biased strains than by unbiased strains) but may be sufficient to maintain haplo-lethals in a polymorphic state for intra-tetrad mating rates near the 0.7 threshold.

Host-parasite systems exist as spatial structures with various degrees of connectivity between populations or communities (Johnson and Boeriijst, 2002). Limited dispersal of hosts and parasites creates various levels of selection and shapes the evolution of host defences and parasite virulence (Boots and Sasaki, 1999, 2000). Our results suggest that antagonistic pleiotropy may be an important feature for the persistence of genetic variation, when acting in combination with populationlevel selection. Such combination of selective forces may be particularly important for the evolution of transmission rates in parasites. Individuals with different transmission rates can coexist in a metapopulation if those that have a lower transmission rate, and hence lower fitness within populations, can also induce a lower extinction rate of the populations where they are prominent, for instance, by allowing their host population to reproduce more or by keeping their hosts alive longer. In addition, pleiotropic advantages associated with the low transmission rate may facilitate the maintenance of polymorphism.

\section{Acknowledgements}

We thank Anne Thomas, Jacqui A Shykoff, Aurélie Garnier, Claire Lavigne and Catherine MontchampMoreau for helpful discussions; Jacqui A Shykoff, Kathryn North, Michel Veuille and anonymous referees for useful comments on the manuscript; Frédéric Austerlitz for suggestions on seeds dispersal modelling, Etienne Klein and Emmanuelle Porcher for advice on statistical analyses.

\section{References}

Antonovics J, Thrall PH, Jarosz AM, Stratton D. (1994). Ecological genetics of metapopulations: the Silene-Ustilago plant-pathogen system. In: Real LA (ed). Ecological Genetics. Princeton University Press: Princeton, New Jersey. pp 146-170.

Antonovics J, O'Keefe K, Hood ME (1998). Theoretical population genetics of mating-type linked haplo-lethal alleles. Int J Plant Sci 159: 192-198.

Baird ML, Garber ED (1979). Genetics of Ustilago violacea. V. Outcrossing and selfing in teliospore inocula. Bot Gaz 140: 89-93.

Boots M, Sasaki A (1999). 'Small worlds' and the evolution of virulence: infection occurs locally and at a distance. Proc $R$ Soc London B 266: 1933-1938.

Boots M, Sasaki A (2000). The evolutionary dynamics of local infection and global reproduction in host-parasite interactions. Ecol Lett 3: 181-185.

Chotivanich K, Udomsangpetch R, Pattanapanyasat K, Chierakul W, Simpson J, Looareesuwan S et al. (2002). Hemoglobin E: a balanced polymorphism protective against high parasitemias and thus severe P. falciparum malaria. Blood 100: 1172-1176.

Couvet D, Ronce O, Gliddon C (1998). The maintenance of nucleocytoplasmic polymorphism in a metapopulation: the case of gynodioecy. Am Nat 152: 59-70.

Curtsinger JW, Service PW, Prout T (1994). Antagonistic pleiotropy, reversal of dominance, and genetic polymorphism. Am Nat 144: 210-228.

Delmotte F, Bucheli E, Shykoff JA (1999). Host and parasite population structure in a natural plant-pathogen system. Heredity 82: 300-308.

Felsenstein J (1976). Theoretical population genetics of variable selection and migration. Ann Rev Genet 10: 253-280.

Garber ED, Baird ML, Weiss LM (1978). Genetics of Ustilago violacea. II. Polymorphism of color and nutritional requirements of sporidia from natural populations. Bot Gaz 139: 261-265. 
Getz WM, Pickering J (1983). Epidemics models: thresholds and population regulation. Am Nat 121: 892-898.

Giraud T (2004). Patterns of within population dispersion and mating of the fungus Microbotryum violaceum parasitising the plant Silene latifolia. Heredity 93: 559-565.

Giraud T, Jonot O, Shykoff JA (2005). Selfing propensity under choice conditions in a parasitic fungus, Microbotryum violaceum, and parameters influencing infection success in artificial inoculations. Int J Plant Sci 166: 649-657.

Giraud T, Jonot O, Shykoff JA (2006). Common sex-linked deleterious alleles in a parasitic fungus alter infection success but show no pleiotropic advantage. J Evol Biol 19: 970-980.

Hedrick PW (1999). Antagonistic pleiotropy and genetic polymorphism: a perspective. Heredity 82: 126-133.

Hood ME (2003). Dynamics of multiple infection and withinhost competition by the anther-smut pathogen. Am Nat 162: 122-133.

Hood ME, Antonovics J (2000). Intratetrad mating, heterozygosity, and the maintenance of deleterious alleles in Microbotryum violaceum (= Ustilago violacea). Heredity 85: 231-241.

Jennersten O (1988). Insect dispersal of fungal disease: effects of Ustilago infection on pollinator attraction in Viscaria vulgaris. Oikos 51: 163-170.

Johnson CR, Boeriijst MC (2002). Selection at the level of the community: the importance of spatial structure. Trends Ecol Evol 17: 83-90.

Kaltz O, Shykoff JA (1997). Sporidial mating-type ratios of teliospores from natural populations of the anther smut fungus Microbotryum (=Ustilago) violaceum. Int J Plant Sci 158: $575-584$.

Kaltz O, Shykoff JA (1999). Selfing propensity of the fungal pathogen Microbotryum violaceum varies across Silene latifolia host plants. J Evol Biol 12: 340-349.

Kisdi E, Geritz SAH (1999). Adaptive dynamics in allele space: evolution of genetic polymorphism by small mutations in a heterogeneous environment. Evolution 53: 993-1008.

Oudemans PV, Alexander HM, Antonovics J, Altizer S, Thrall PH, Rose L (1998). The distribution of mating-type bias in natural populations of the anther-smut Ustilago violacea on Silene alba in Virginia. Mycologia 90: 372-381.
Prout T, Savolainen O (1996). Genotype-by-environment interaction is not sufficient to maintain variation: levene and the leafhopper. Am Nat 148: 930-936.

Richman A (2000). Evolution of balanced genetic polymorphism. Mol Ecol 9: 1953-1963.

Roche BM, Alexander HH, Maltby AD (1995). Dispersal and disease gradients of anther-smut infection of Silene alba at different life stages. Ecology 76: 1863-1871.

Rose MR (1982). Antagonistic pleiotropy, dominance, and genetic variation. Heredity 48: 63-78.

Rose MR, Charlesworth B (1981). Genetics of life-history in Drosophila melanogaster. I. Sib-analysis of adult females. Genetics 97: 173-186.

Shykoff JA, Bucheli E (1995). Pollinator visitation patterns, floral rewards and the probability of transmission of Microbotryum violaceum, a venereal disease plant. J Ecol 83: 189-198.

Tellier A, Villaréal LMMA, Giraud T (2005). Maintenance of sexlinked deleterious alleles by selfing and group selection in metapopulations of the phytopathogenic fungus Microbotryum violaceum. Am Nat 165: 577-589.

Thomas A, Shykoff JA, Jonot O, Giraud T (2003). Sex-ratio bias in populations of the phytopathogenic fungus Microbotryum violaceum from several host species. Int I Plant Sci 164: 641-647.

Thrall PH, Biere A, Antonovics J (1993). Plant-life history and disease susceptibility - the occurrence of Ustilago violacea on different species within the caryophyllaceae. J Ecol 81: 489-498.

Thrall PH, Biere A, Uyenoyama K (1995). Frequency-dependant disease transmission and the dynamics of the Silene-Ustilago host-pathogen system. Am Nat 145: 43-62.

Thrall PH, Jarosz AM (1994a). Host-pathogen dynamics in experimental populations of Silene alba and Ustilago violacea. I. Ecological and genetic determinants of disease spread. J Ecol 82: 549-559.

Thrall PH, Jarosz AM (1994b). Host-pathogen dynamics in experimental populations of Silene alba and Ustilago violacea. I. Ecological and genetic determinants of disease spread. J Ecol 82: 549-559.

Zar J (1984). Biostatistical Analysis. Prentice Hall: London, UK. 